\title{
RETRACTED ARTICLE: Neural auditory intensity discrimination during naturalistic listening: a brain decoding study
}

\author{
Xintao $\mathrm{Hu}^{1} \cdot$ Lei Guo ${ }^{1} \cdot$ Junwei Han $^{1} \cdot$ Christine Cong Guo ${ }^{2}$
}

Received: 12 May 2016/ Accepted: 11 October 2016/Published online: 21 October 2016

(c) Springer-Verlag Berlin Heidelberg 2016

This article has been retracted at the request of the Editorsin-Chief and the Publisher due to extensive overlap with a previously published article [1]. One of the co-authors, Christine Cong Guo, states that she was not aware that overlapping findings had already been submitted and published in Brain Imaging and Behavior. None of the authors agree to this retraction. The online version of this article contains the full text of the retracted article as electronic supplementary material.

\section{Reference}

1. Hu X, Guo L, Han J, Liu T (2017) Decoding power-spectral profiles from FMRI brain activities during naturalistic auditory experience. Brain Imaging Behav 11:253-263. doi:10.1007/ s11682-016-9515-8

Electronic supplementary material The online version of this article (doi:10.1007/s00429-016-1324-8) contains supplementary material, which is available to authorized users.

Junwei Han

jhan@nwpu.edu.cn

1 School of Automation, Northwestern Polytechnical University, Xi'an, China

2 QIMR Berghofer Medical Research Institute, Herston, QLD, Australia 